\title{
Summary and Reflections ${ }^{1}$
}

\author{
Robert D. Mathieu \\ Department of Astronomy, University of Wisconsin, Madison WI 53706
}

Let me begin by taking a very broad historical perspective on the study of premain-sequence binaries. You have seen reference several times at this meeting to that first paper on T Tauri binaries by Joy \& Van Biesbroeck (1944). Following that paper there followed a long period that might be called the Decades of Ignorance. I refer here to a period of time in the study of star formation where we largely ignored the fact that we knew that most stars were binaries. The high frequency of binaries among stars is something that we've known for a very long time, well over a century. Indeed, looking back, it is remarkable that the paper in 1982 by Mel Dyck and his collaborators identifying T Tauri as a binary caused such a stir. This paper is sometimes cited as the birth of modern research into young binaries. But how many of our colleagues in the realm of main-sequence binaries had already told us over and over again that most of these young stars had to be binaries? Of course, the reason this important paper caused much ado was in part because $\mathrm{T}$ Tauri is the very prototype star of star-formation study.

This discovery of T Tauri's binary nature was followed by a period that I'll call the Decade of Acknowledgment. This was a very enjoyable time in my life, because, as I think Hans Zinnecker will affirm, at most every star-formation meeting either Mike Simon, Bo Reipurth, Hans, or myself was invited to give the one and only binary talk. Occasionally they would even let Hans and me both talk! We were a very small circle, and we saw each other more often than I think we do now.

There was a change of state in in the early '90s linked to the advent of large-scale high-resolution infrared surveys. The dissertation of Andrea Ghez, the wonderful work by Christoph Leinert and his collaborators in Taurus, the imaging of southern star-forming regions by Bo and Hans, and several other papers, almost simultaneously drove home to the field of star formation what I think we already knew (or should have known!), and that was that most pre-main-sequence stars were binaries. This was a very powerful time, and we rapidly evolved into what I would call the Decade of Acceptance as starformation meetings began to increase their emphasis on young binaries. Which of course, has led to this meeting and what will presumably be the Millennium of Dominance. (Laughter)

I think we will have reached the point of true awareness when the agenda of the next star-formation meeting is very similar to the agenda of this meeting. I've been saying for years that binary-star formation is the primary branch of the star-formation process, but at this meeting Richard Larson took a dramatic

\footnotetext{
${ }^{1}$ This paper is a verbatim transcription of the presented talk, with minor editing.
} 
conceptual leap in suggesting that perhaps we don't need a single-star formation process at all. We shall see where he lands!

Now, with this historical perspective in mind, I'd like to make a comment about the state of our field. I apologize to all the historians and philosophers of science, because these comments are grossly simplistic, but I hope they may be of value nonetheless. In terms of the progress of a field, I think it's fair to say that there often is a discovery result, or several results, that give birth to the field, followed soon thereafter by lamentations of having too few data. And, if the discovery is truly important, there next follows lamentations of having too many data and consequent confusion! I think this is the state that we are in right now. Much of the confusion that we've seen this week is a wonderful thing, because it derives from a great deal of superb data. At the same time we do need to recognize that our field is young and that it is going to require long hard work to move to a place of understanding. I use as my exemplar the study of the initial mass function, which came into its own in the late '50s. Much of the ' $60 \mathrm{~s}$, if you look at the literature, was spent examining clusters and the field to assess whether or not the initial mass function was "universal" (much akin to our present efforts to see if binary frequency is the same across regions). Many studies were done, and with many twists and turns regarding whether the initial mass function was or was not universal. And after some 30 more years, while I do think we have a much clearer understanding of the initial mass function, it is fair to say that there are still many basic questions in that subject without satisfactory answers, including the issue of universality. Learning from our predecessors, we should be wary of putting too much stock in the recent wanderings of our own observational efforts. A smoothing interval of at least five to ten years is invaluable so that we can identify what is secure and what is transient. Aristotle and his "collaborators" were in error for 2000 years; we have been working on this subject for perhaps 10 years. Let us not take ourselves too seriously yet!

With those thoughts in mind, let me address some specific issues of this meeting. Formation mechanisms are central, of course. Fortunately Cathie Clark has already done a spectacular job of summarizing the entire subject of formation mechanisms. Cathie's comments can be summarized by her observation that all the data contradict the models and all the data contradict each other. (Laughter) Again, that period of confusion that we're in right now. There's nothing wrong with that, it is part of the process.

In respect to formation mechanisms, Alan Boss came up to me and said, "Bob, this is a seminal meeting, because I think this is going to be the meeting that is pointed to as the moment where it was realized that fragmentation is clearly the answer." Alan's comment rather caught me by surprise, for while I fully agree that fragmentation is the answer, I'm not entirely sure what is the question! (Laughter) In the 1996 NATO meeting in Cambridge, the hypothesis of capture formation in its classic guise was finally put to rest. Similarly, the idea of fission of a single star, with all due respect to Joel Tohline, was also seen to have at least one foot in the grave. The issues that were discussed in Cambridge, and I think still need to be discussed, are not whether fragmentation happens but whether it is fragmentation of protostellar clouds, fragmentation of 
disks, fragmentation directly into two bound stars, fragmentation into small-N systems with consequent evolution, and so on. Or whether, in fact, the primary fragmentation mode is to clusters rather than binaries, with subsequent evolution involving dynamical processes that are reminiscent of those that have been invoked for capture. Indeed, given that small-N systems and clusters clearly form, there is no doubt in my mind that there is "capture", but it's not in the classic sense. It is the dynamical formation of binary systems subsequent to fragmentation, and may lead to very different evolutionary paths for fragmented systems.

As Ian Bonnell warned us, I think all of the fragmentation processes are a good deal more complicated than we would like to have it. Frank Shu said that the worst situation is magnetohydrodynamic turbulence with radiation. Of course, that is what we have. An interesting idea that has been mentioned several times at this meeting is the possibility that it is a magnetic phase change that actually leads to fragmentation. Finally, I remind you once again that the formation of close binaries remains wholly unsolved, by fragmentation or any other process. Having such a major subset of objects are understood is an important statement about the youth of the field.

Let me go on a little bit further with my theoretical perspectives. Richard told us that there is no standard model for binary formation. A daunting statement but perhaps true, especially when you look at it in the light of the very broad distributions of the key parameters. Distributions in period, distributions in secondary mass functions, distributions in eccentricity. Quite possibly the seminal idea that may come out of this meeting is that binary formation is in fact a synthesis of many processes. And the essential interface between theory and observation may be the assessment of which processes are contributing at what fractions to the entire binary population. This perspective is fundamentally different from our previous approach of making predictions from each process and then checking off boxes of "yea" or "nay" with respect to observations in an attempt to determine which process is "correct". I think this is unlikely to be productive for us. I think, as Cathie said, most if not all of these processes are going on, and probably more we haven't thought of. The issue is how to synthesize them to produce the young binary populations that we observe.

Let me now turn to new insights from observations. Main-sequence binary populations act as the boundary condition for our field, and I think our compatriots who are working on these populations have provided us with significant new results. First, Michel Mayor reported that in their major study of the $\mathrm{M}$ dwarfs, the M-dwarf binary population seems to be remarkably similar to the G-dwarf population. This lack of mass dependence among lower mass stars would seem to have important implications for the formation process. At the same time I think it is becoming clear that there is a dependence of the binary frequency on mass as one goes to more massive stars. Indeed, the most striking observational result that I saw at this meeting was perhaps the most understated. Jean-Claude Mermilliod told us that among the O-type stars that he'd been studying in clusters, essentially every single one - I exaggerate just a bit - had a companion with a period of less than eight days. That's absolutely shocking! This morning, I was having breakfast with Matt Holman and I 
asked him, "What does it mean when 14 out of 15 stars are very short period binaries?" Matt very wisely said, "It means that one of them lost their companion." (Laughter) When combined with other reports at this meeting of rich binary populations over a range of separations among OB stars, it really makes me wonder what is going on in those O-stars. Perhaps the answer is linked to formation of $\mathrm{O}$ stars through collisions, but I am not convinced - particularly with respect to the high frequency of wide companions. But most assuredly I am more intrigued by these massive stars than I was when I walked into this room a week ago.

Given that most pre-main-sequence binary populations have been surveyed at moderate to wide separations, I think it is critical that we reassess the mainsequence wide-binary distribution in the field. As Andrea said, we must compare apples with apples. Remember, most of the statements that Michel was making about relative binary populations were made in the spectroscopic domain. Recall also that in the classic Duquennoy \& Mayor paper, the only new data were spectroscopic. For the wide binaries Duquennoy \& Mayor used information in the literature, analyzed it as best possible, and then derived the G-dwarf binary frequency distribution as a function of period. How many times during this meeting have we seen those results projected on the screen? If those results really are that important, and I would posit to you that they are, it seems that we should be providing as much attention on improving those data as we are on improving data in the pre-main-sequence domain. Indeed, from a purely statistical point of view, we're reaching the point where the statistical uncertainty in the main-sequence binary frequencies are comparable to those of the pre-main-sequence binary frequencies. When Michel and Dave Latham tell you that there are new very large samples being studied, I believe they're mostly being studied spectroscopically. I certainly hope that the new interferometers such as CHARA will be observing these same stellar samples. Alas, as Andrei Tokovinin told us, in no case can we be sure that we actually know all the components of the system.

Turning now to observations of pre-main-sequence binary populations, the secondary mass distribution is proving absolutely critical and absolutely difficult. With all due respect to those who are working very hard on this problem, I don't think we've got it yet. So I think its premature to summarize our findings here, but I do want to encourage those who have taken up this challenge and to endorse the importance of their work. This is a difficult problem that must be done very, very well.

Nonetheless, I am very intrigued by this brown dwarf desert. It seems to be gaining more and more validity as we get more data. The reason this is intriguing lies in a simple question which maybe you could muse about if you haven't already: why should the process that determines secondary masses know anything about the hydrogen burning limit? Hydrogen burning is a phenomenon that is going to occur much, much later in the evolution of the star. Why should the fragmentation process and the subsequent evolution know anything about 0.08 solar masses?

In terms of pre-main-sequence binary frequencies by region or age, we have accumulated a large number of observations and are now challenged with making sense of them. It seems that the young open clusters are coming in with 
binary frequencies more or less similar to the field binary frequency. However I'm still not clear about what different star-forming regions are telling us, or indeed whether the measured differences are real. Certainly Taurus has a remarkably high binary frequency; as Pat Hartigan quipped, "It would be nice if at least some of these stars weren't binaries". (Laughter) With the many excellent studies underway, I think we can hope to clarify the dependence of binary frequency on physical conditions of star-forming regions in the next few years.

Binary environments are fascinating places in the Universe. Karl Stapelfeldt made a very insightful comment when he said that $T$ Tauri is the prototype of confusion. We always have a tendency to try to simplify for understanding, and yet when we actually look at the objects it is rather striking that they're very complex. In my opinion, at this meeting the survival of circumstellar disks has come to the fore as a central issue. Disk truncation is secure; that's a quote from Ann Dutrey, and I agree with her. I also think that as a consequence replenishment of circumstellar disks must happen, and it is a fascinating question whether the mechanism is accretion streams from circumbinary disks or infall or both.

More broadly, I'd like to raise a very large issue. Circumstellar disk physics has developed extensively over the last decade in the context of single stars. For example, when Bo shows us these absolutely gorgeous pictures of jets - and I'm really excited to show these to you; I've been doing astronomy since I was eight years old because of magnificent pictures like these - we must recognize that the present theories on how to drive jets are primarily being developed in the context of single disks around single stars. Similarly for the many other diskrelated processes of early stellar evolution - accretion and winds and radiation transfer and and disk locking. All of these fundamental components of our starformation model need to be reevaluated coming out of this meeting, because few if any of the stars against which the theories have been tested are single stars. And in many cases, where the circumstellar disks are severely truncated for example, it's not at all obvious to me that there is the material there to drive the proposed astrophysical processes. We need to seriously reassess much of the single star - disk paradigm in the context that most of those single stars are binaries.

Now, with respect to pre-main-sequence stellar masses, what can I say? All I have is this feeling of warmth - we are actually displaying pre-main-sequence evolutionary tracks with measured masses! Its going to be one of the key breakthroughs of this meeting that there are now masses with which to test the models. Please indulge me in making a couple points with regard to the strengths of the different methods of determining stellar masses. Eclipsing binaries are rich mines of information. You obtain two masses, two radii, one effective temperature (I emphasize that you still have to get an effective temperature from somewhere else) and the distance. Mike raised a very important point, though, which is the issue of close binary evolution. Eclipsing binaries are almost always relatively close, usually with periods of less than ten days, sometimes only a couple of days. The stars could not have fit into those orbits when they were protostars, or indeed even when they were at the stellar birthline entering the pre-main-sequence phase of evolution. So there is a very real question of whether the interior evolution of such stars allows them to be appropriate test cases for 
stellar evolution models. Astrometric-spectroscopic orbits, which I think are the wave of the next decade, provide two masses and distance, without this annoyance of close evolution. These really are superb targets for study. Disk rotation curves give you one mass, and are dependent upon independent derivations of distance.

In the light of the tremendous recent progress in measuring masses of premain-sequence stars, I suggest that the stellar mass, oddly enough, is not really the limiting parameter. Suppose you choose a star, any star - BP Tau, for instance - and I tell you that the mass of that star is 0.9378 solar masses. Remarkable accuracy, eh? It still does you no good unless you can compare that star to the tracks with comparable accuracy. In particular, in the domain of the Hayashi tracks you need to be able to determine accurately the effective temperature of the star. It certainly helps to also determine accurately the luminosity of that star. And so while we've been lamenting for years that we need mass measurements, and we do, now that we have them we have to be sure that we are comparing the stars to the tracks with the same level of accuracy as the masses. And that is one of the advantages of eclipsing binaries because the radii give you a great deal of help in that.

On the theoretical side of pre-main-sequence stellar evolution, I have to say that I thoroughly enjoyed the "debate" yesterday between Isabelle Baraffe and Günther Wuchterl. It was one of the highpoints of the conference for me, and I would like to thank both of the debaters. This figure of Günther I find very important. [See Figure 1 of Wuchterl article in these proceedings.] It very nicely puts in context the debate that we've been having of late over the correct pre-main-sequence tracks. While this debate is vital and necessary, it represents nonetheless only a portion of the entire early evolution of the star, and a portion that may not be independent of what has gone before. At some level it must be true that we have to move toward including the entire pre-main-sequence evolutionary process in our discussion of early evolution.

Late last night, after that wonderful dinner, I started planning the program for our next meeting ... which will be in Madison ... in 2010 ... in the summer! What will be the new important subjects at that meeting?

In my Annual Reviews article in 1994 I wrote that we had just been through a decade of discovery for $\mathrm{T}$ Tauri binaries, and that we were moving into the decade of discovery for protobinaries. We are moving strongly in that direction, but a half-decade later I think we are still only on the verge of that age of protobinary discovery. By $2010 \mathrm{I}$ anticipate that we will indeed be in a position to have a really substantive discussion of protobinaries.

Another critical issue is going to be the orientation of angular momentum, which has to be central to the process of binary formation. At this meeting we have started to see new polarization results, which have suggested the really intriguing idea that the relative orientations of disks in wide binaries are not random. And of course Francois Menard and Karl showed such wonderful HST images of misaligned discs that I think there can be little doubt that at least in wide binaries disks are not aligned. The issue is more whether they are randomly oriented or whether there remains some knowledge in the disks of each other or of the orbital angular momentum, whether there are correlations present in the 
observations. And, being among the authors of one of the polarization papers, I want to remind you of my opening discussion. We are not even in the period of confusion yet; we're in the period of a few results. Don't take results too seriously, yet, but be excited by them and pursue them so that ten years from now we can begin to understand the evolution of angular momentum in binary formation.

In the next decade we must begin to think about young binaries as evolving systems. Numerous processes lead to the evolution of binary-disk systems, and quite rapidly compared to the stellar evolution timescales. Whether as a result of Matthew Bate's infall or Pavel Artymowicz and Steve Lubow's accretion streams or gravitational torques from circumbinary disks or tidal dissipation or all of these processes and more, the orbits and disks of young binaries change during the pre-main-sequence phase. Furthermore, as a direct consequence of the misalignment of angular momentum vectors, this evolution is occurring in three dimensions. Caroline Terquem has been wisely counseling us to consider both the dynamical and radiative consequences of warps in disks.

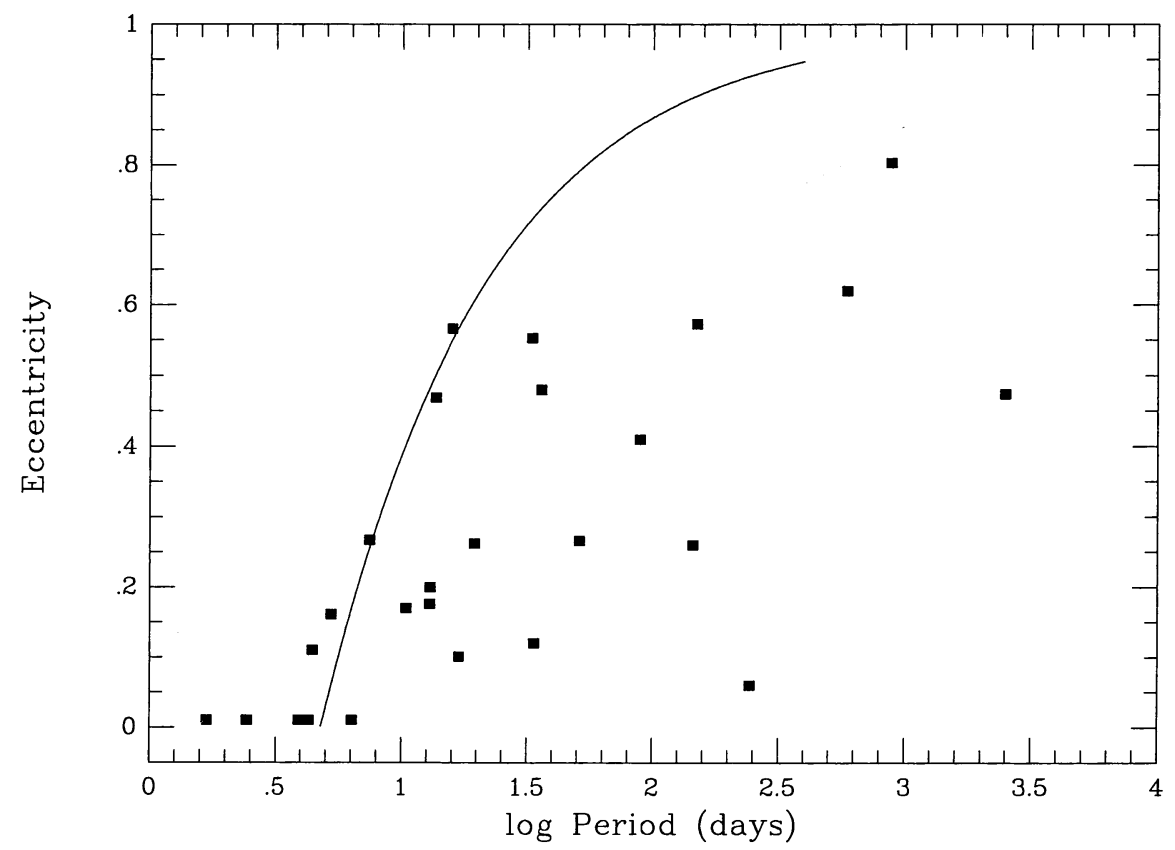

Figure 1. The pre-main-sequence eccentricity distribution and the curve of constant periastron separation $\mathrm{R}_{\text {peri }}=15 \mathrm{R}_{\odot}$ (Mathieu 1992).

Finally, I will go out on a limb and predict that the subject of pre-main sequence contact binaries will be born in the next decade. I don't know quite why we haven't found them yet; perhaps it is that we don't know what they look like. But I am confident that they are out there. This is the pre-mainsequence eccentricity-log period distribution that I created for the Bettmeralp meeting in 1992. [Figure 1] I included on this figure a curve of constant periastron 
separation at $15 \mathrm{R}_{\odot}$ for two solar-mass stars, recognizing that solar-mass stars at the stellar birthline have radii of approximately $5 \mathrm{R}_{\odot}$. The curve is very similar to the upper envelope of the eccentricity-period distribution that Pavel Kroupa was also pointing out earlier. Essentially the curve is showing us that much of the triangular "exclusion" region in the upper left of the figure would include binaries with periastron separations of less than a couple stellar radii at the stellar birthline. To me, the existence of the observed envelope suggesting a lower limit on binary separations combined with the likelihood of orbital migration, especially at early ages when circumbinary disks are massive strongly hints that there may be binaries formed which evolve into contact systems. Their evolution at that point is not clear - merger perhaps - but evidently they disappear from this diagram. Hence my prediction - and indeed my hope, for it would be fun that the discovery of contact binaries will be one of the fascinating new subjects of the next meeting.

Adding to this list the topics of binary population synthesis, the role of magnetic fields in fragmentation, and results from the wonderful new technologies that will provide super-high angular resolution, and here we have a first draft of the agenda for our next meeting in 2010 !

I would like to close with this figure created by José Torrelles in which he presented a cartoon model of the seminal object L1551 IRS5. [See Figure 2 in Torrelles et al. article in these proceedings.] As we reconsider this model, it becomes so evident that as a world we need to work together to solve the challenges of the Universe. This cartoon blends work of scientists in from North America, scientists in Asia, scientists in Europe, and scientists in Latin America. The findings from these diverse origins fit together in a vital way to give us deeper insight into this beautiful young binary. Equally importantly, our mutual and shared desire to understand brings us together and gives us deeper insight into each other as well. It is truly wonderful that in this beautiful city of Potsdam, so close to the former border between East and West Germany and the site of so many tensions not long ago, we can all come together to reaffirm our unity as astronomers and as a people of the world.

And so I will end this last talk of IAU Symposium 200 "The Formation of Binary Stars". Thank you!

\section{References}

Mathieu, R.D. 1992, in Binaries as Tracers of Stellar Formation, ed. A. Duquennoy, M. Mayor, Cambridge University Press, 155 


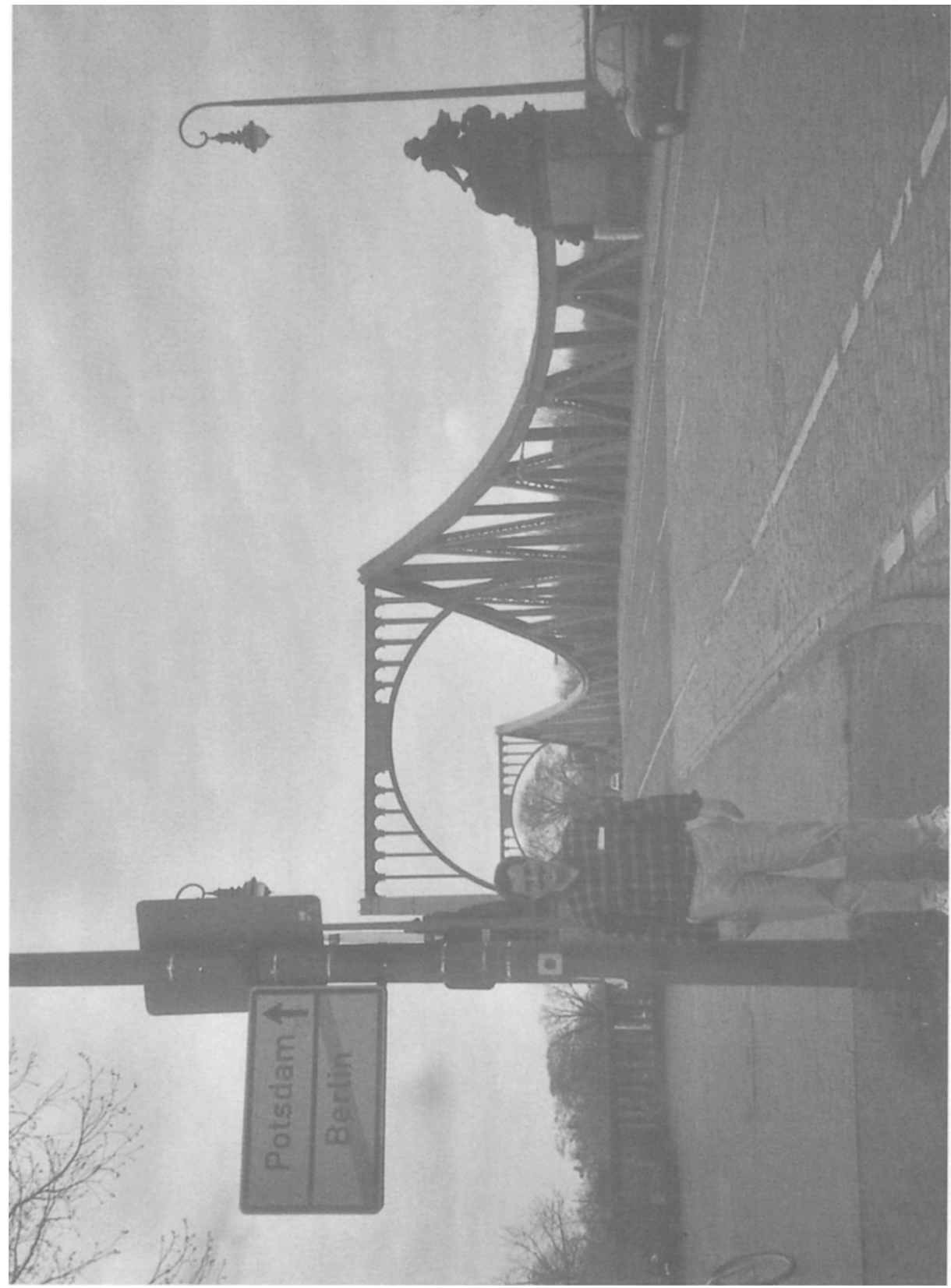

Bob leaving. 